\title{
Protected silver coatings for reflectors
}

Stefan Schwinde*a, Mark Schürmann ${ }^{a}$, Ralph Schlegela, Jan Kinast ${ }^{a}$, Reinhold J. Dorn ${ }^{b}$, Jean Louis Lizon ${ }^{b}$, Sebastien Tordo ${ }^{b}$, Norbert Kaiser ${ }^{a}$

aFraunhofer Institute for Applied Optics and Precision Engineering IOF ${ }^{b}$ European Southern Observatory ESO

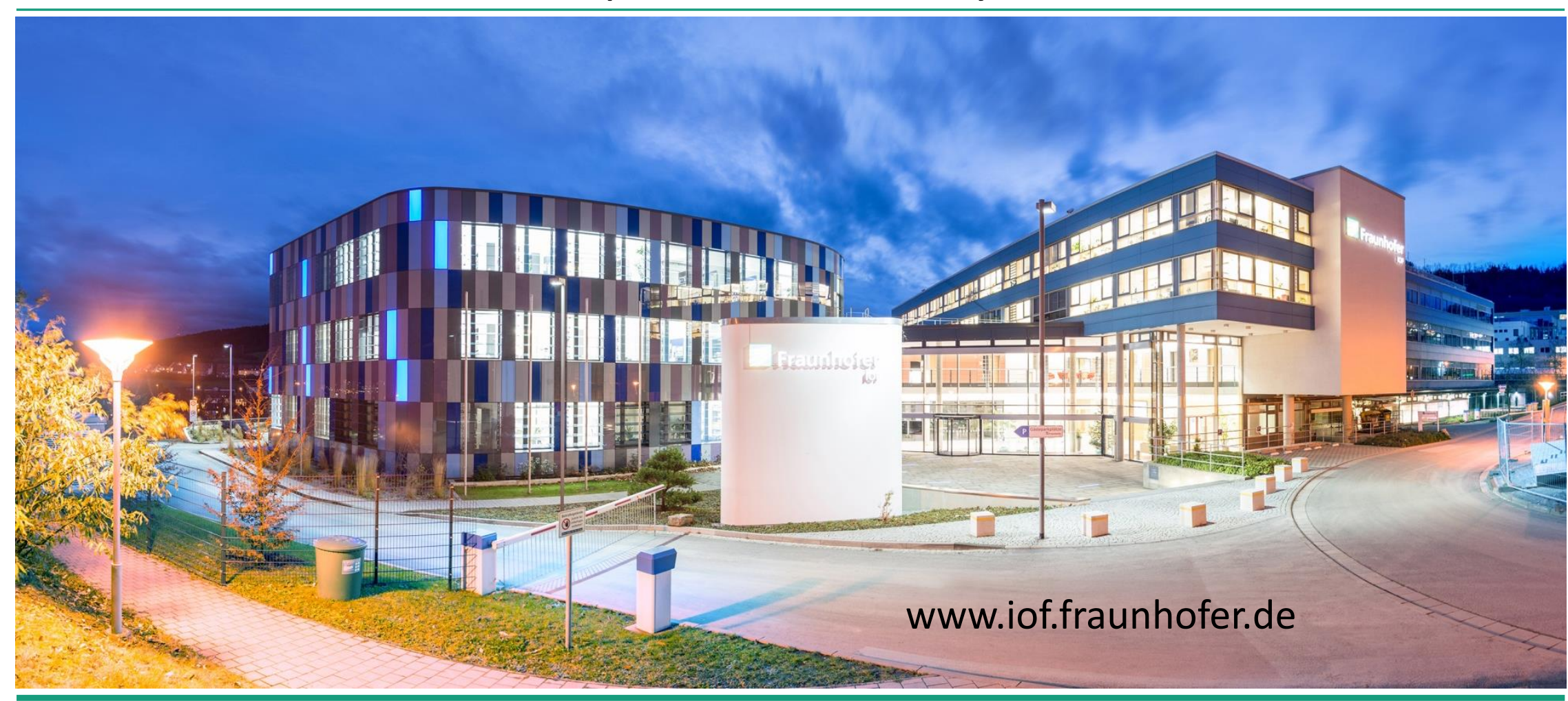




\section{Introduction and Background}

\section{CRIRES + -}

- Crires ${ }^{+}$Upgrade of the CRIRES instrument at the Very Large Telescopes

- IR spectrograph

Research foci: e.g. Investigation of exoplanets

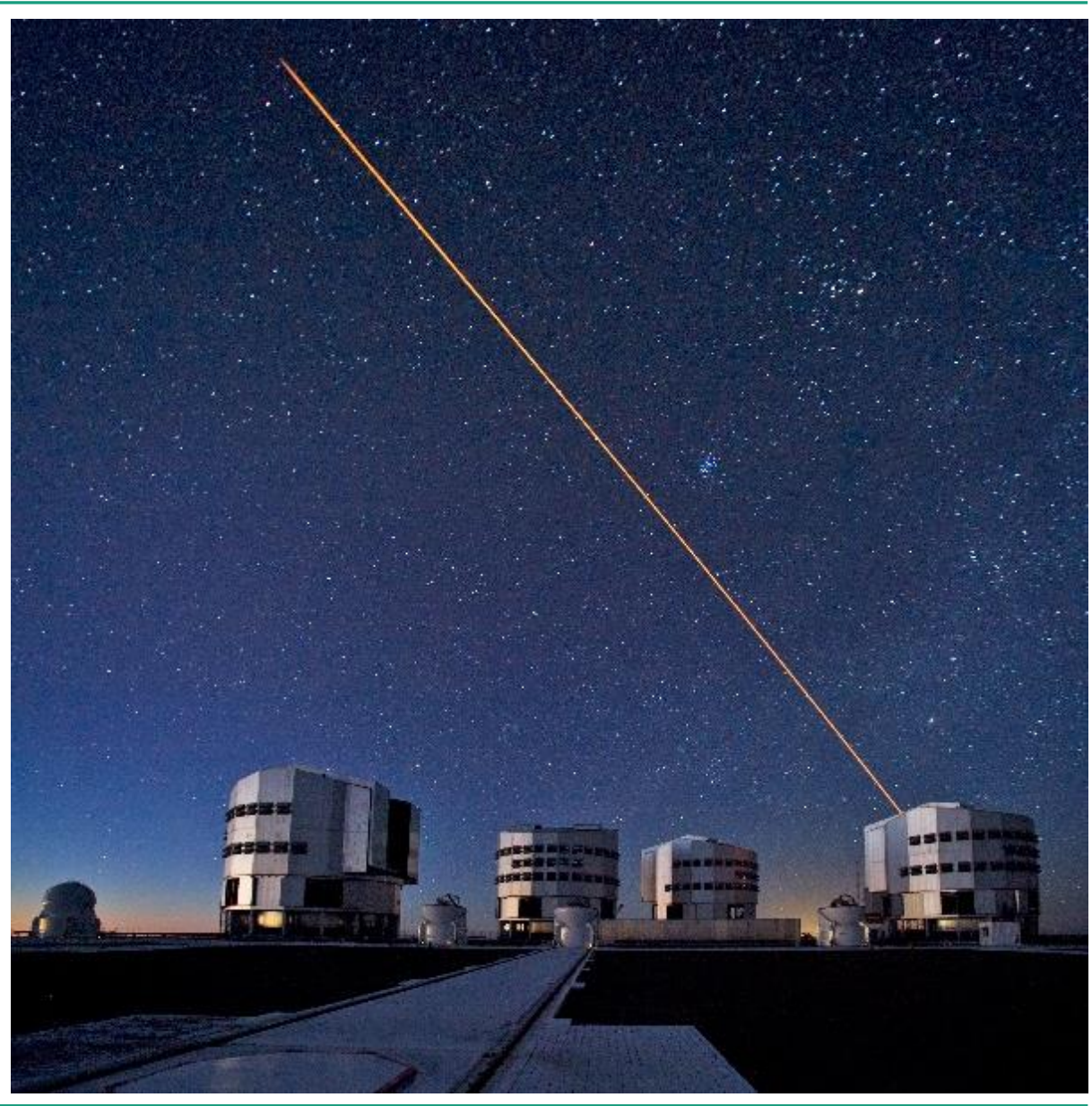




\section{Introduction and Background}

\section{CRIRES+)}

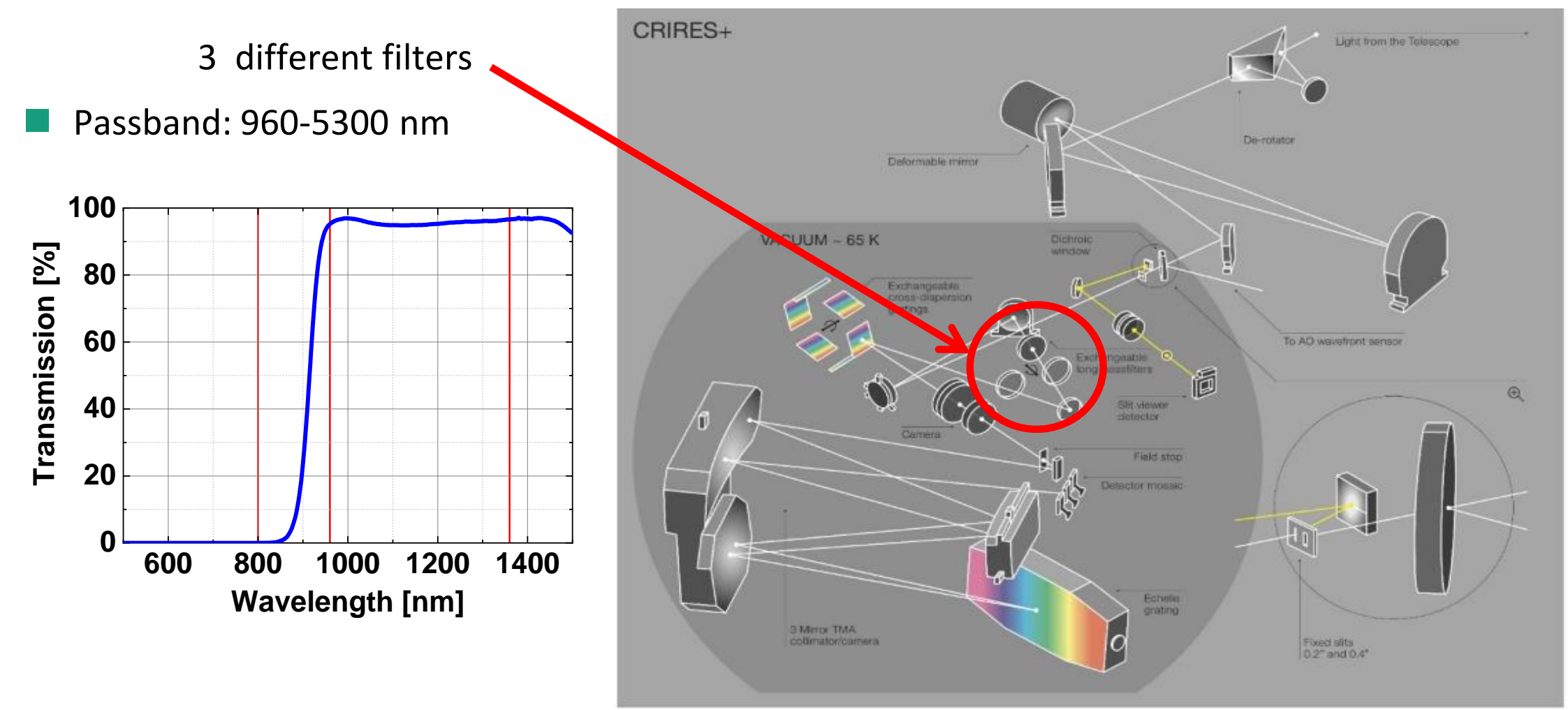




\section{Introduction and Background}

\section{CRIRES+)}

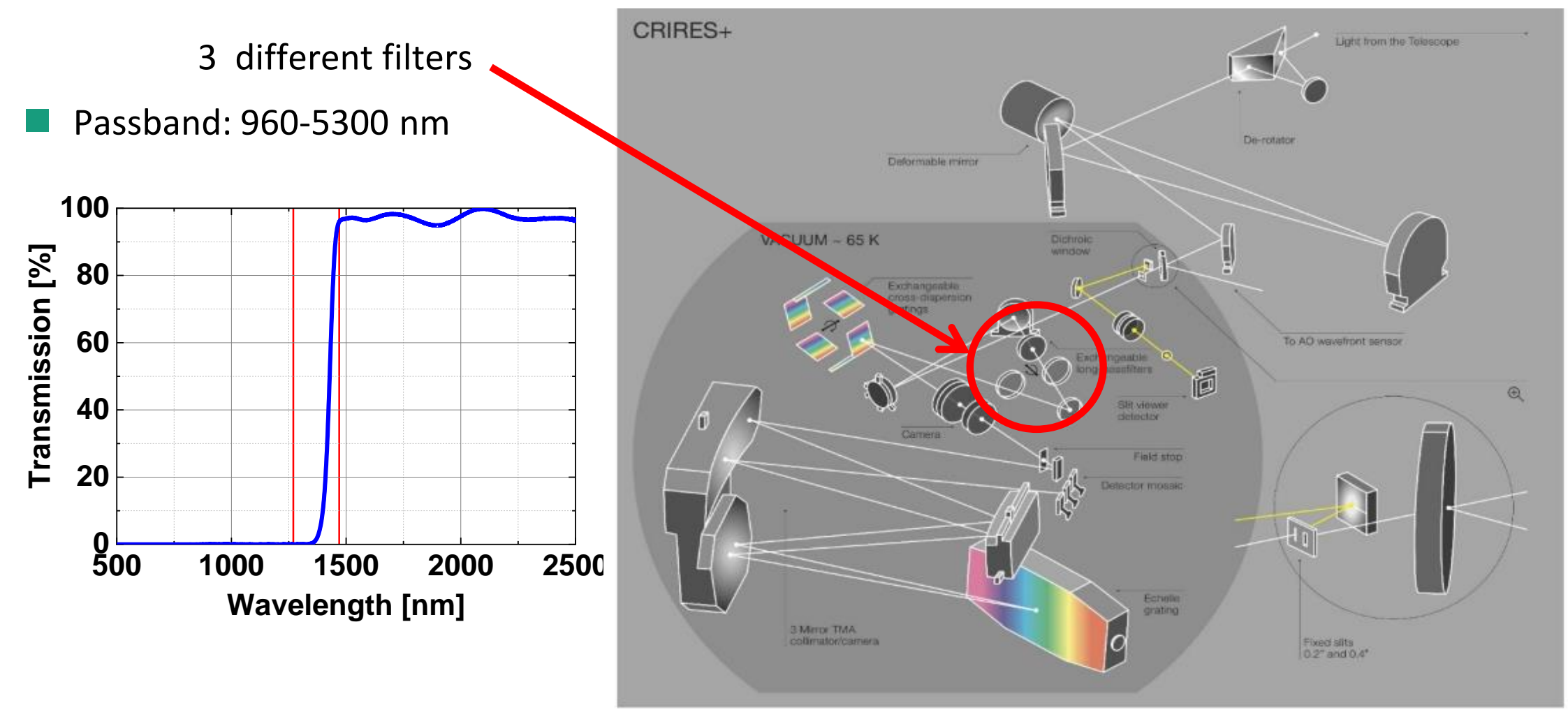




\section{Introduction and Background}

\section{CRIRES+)}

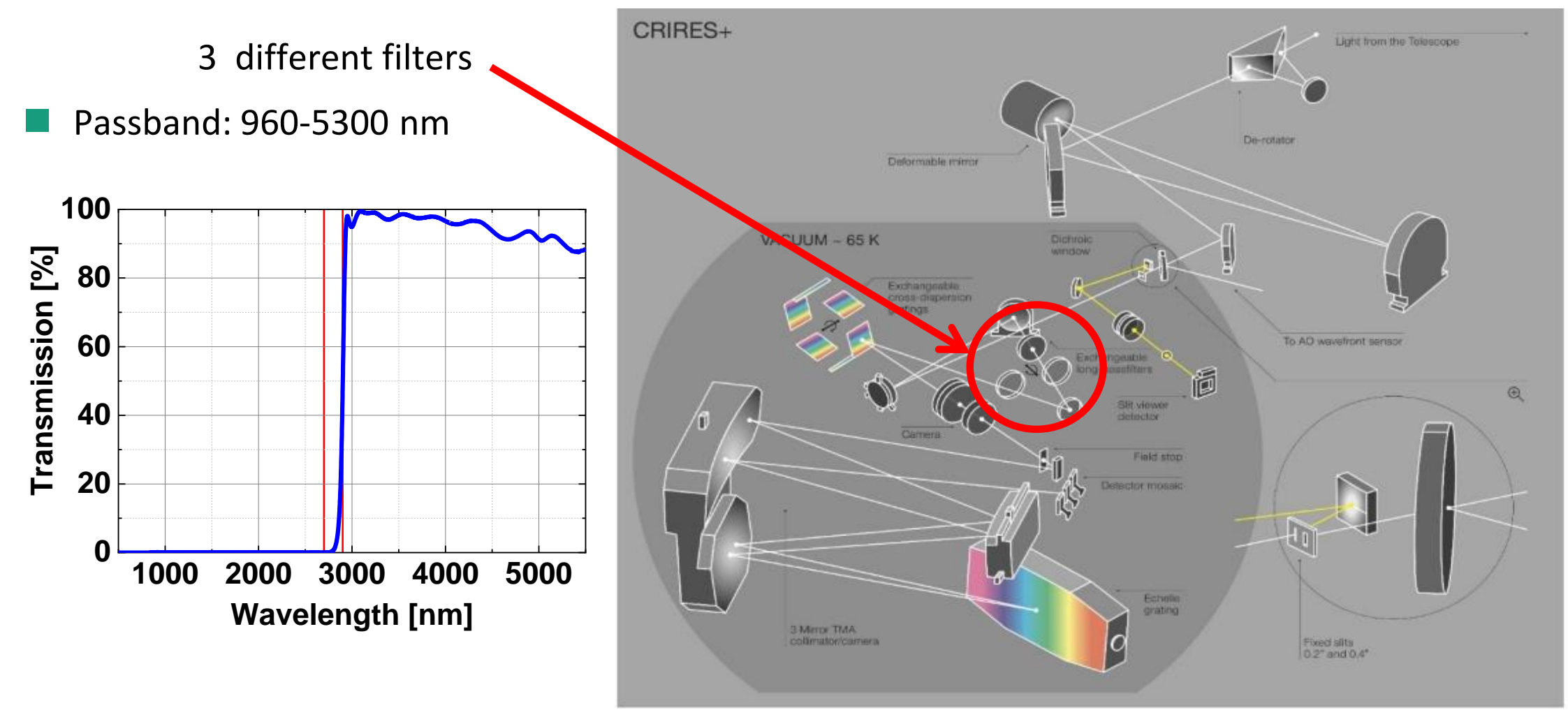




\section{Introduction and Background}

CRIRES+)

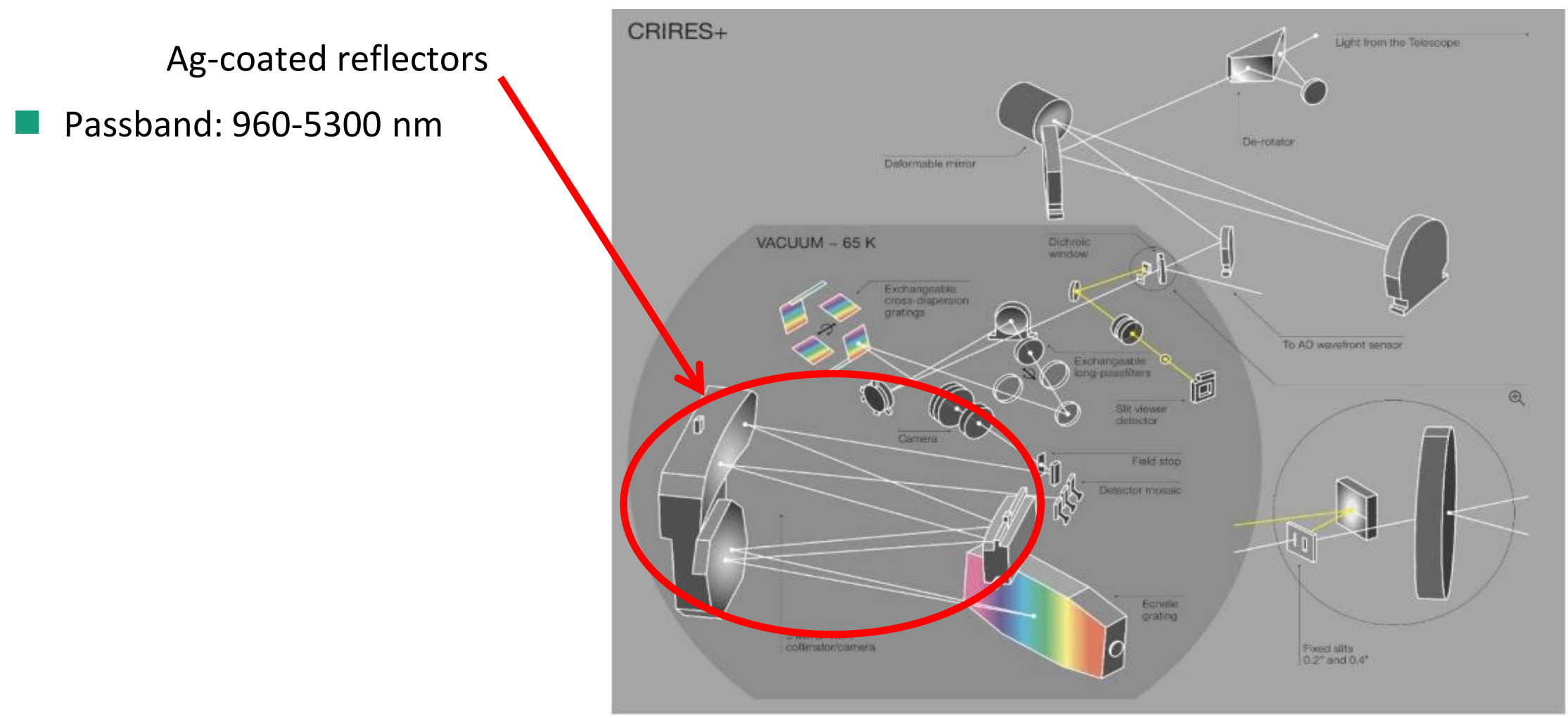




\section{Introduction and Background}

Why to talk about coatings for ground based telescopes on a conference for space optics?

- Coatings from IOF are used for space based applications: e.g. RapidEye, EnMap, Desis,

and for ground based application like e.g. for $\operatorname{Crires}^{+}$(VLT).

Space based applications

specifics: radiation, reduction of mass, ....

n commonalities with ground based applications:

- long period between coating and application (transport, assembling and storage)

$\rightarrow$ risk of contamination

risk management: options for cleaning and refurbishment

- Handling of dielectric coatings is less challenging than handling Ag-based coatings 


\section{Ag-based Coatings (Optical Performance)}

$\mathrm{Ag}$ has the highest reflectivity of all metals

- Reflectivity of unprotected Ag decreases if exposed to ambient condition
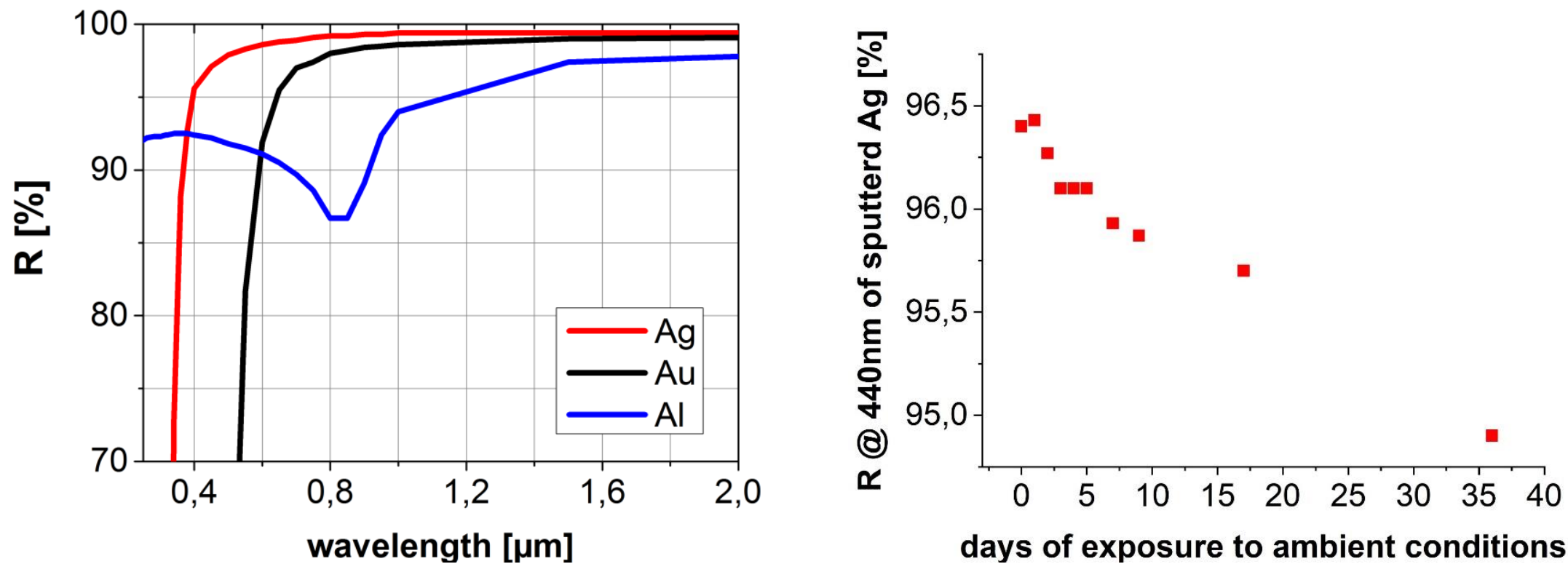


\section{Ag-based Coatings (Protection)}

- Protected Ag to avoid a decrease of reflectivity:

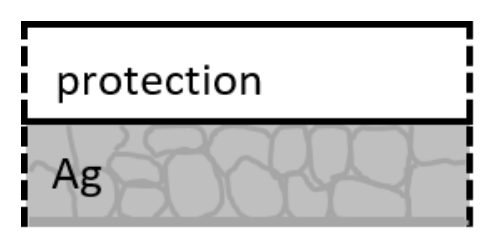




\section{Ag-based Coatings (Protection)}

Protected Ag to avoid a decrease of reflectivity:

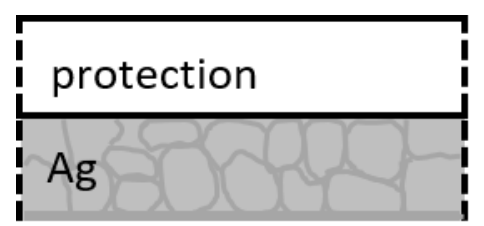

Contamination on the protection [1]:
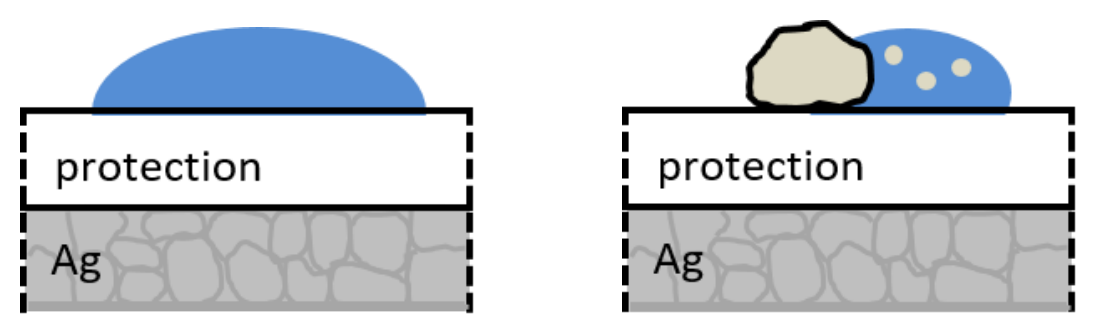


\section{Ag-based Coatings (Protection)}

- Protected Ag to avoid a decrease of reflectivity:

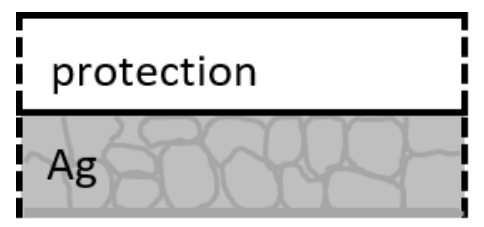

Contamination on the protection [1]:

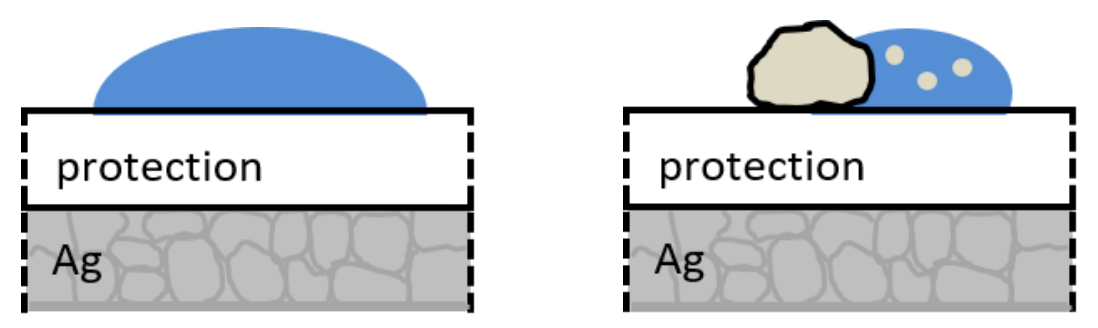

- Risk of buckling delamination due to compressive stress [2]:

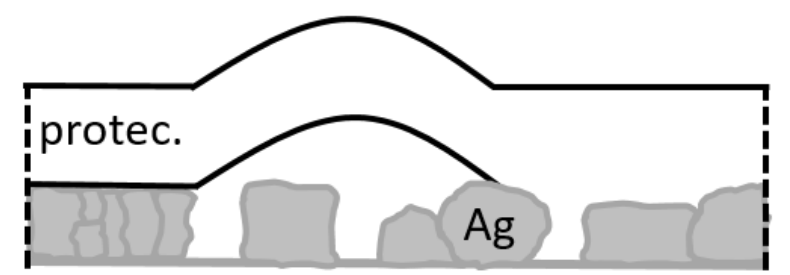

- Protection: high resistance to aqueous solutions and a low compressive stress is required 


\section{Ag-based Coatings (Protection)}

- Protection: high resistance to aqueous solutions and a low compressive stress is required

- Reactive sputtered $\mathrm{Al}_{2} \mathrm{O}_{3}, \mathrm{SiO}_{2}, \mathrm{Si}_{3} \mathbf{N}_{4}$

- Influence of gas supply

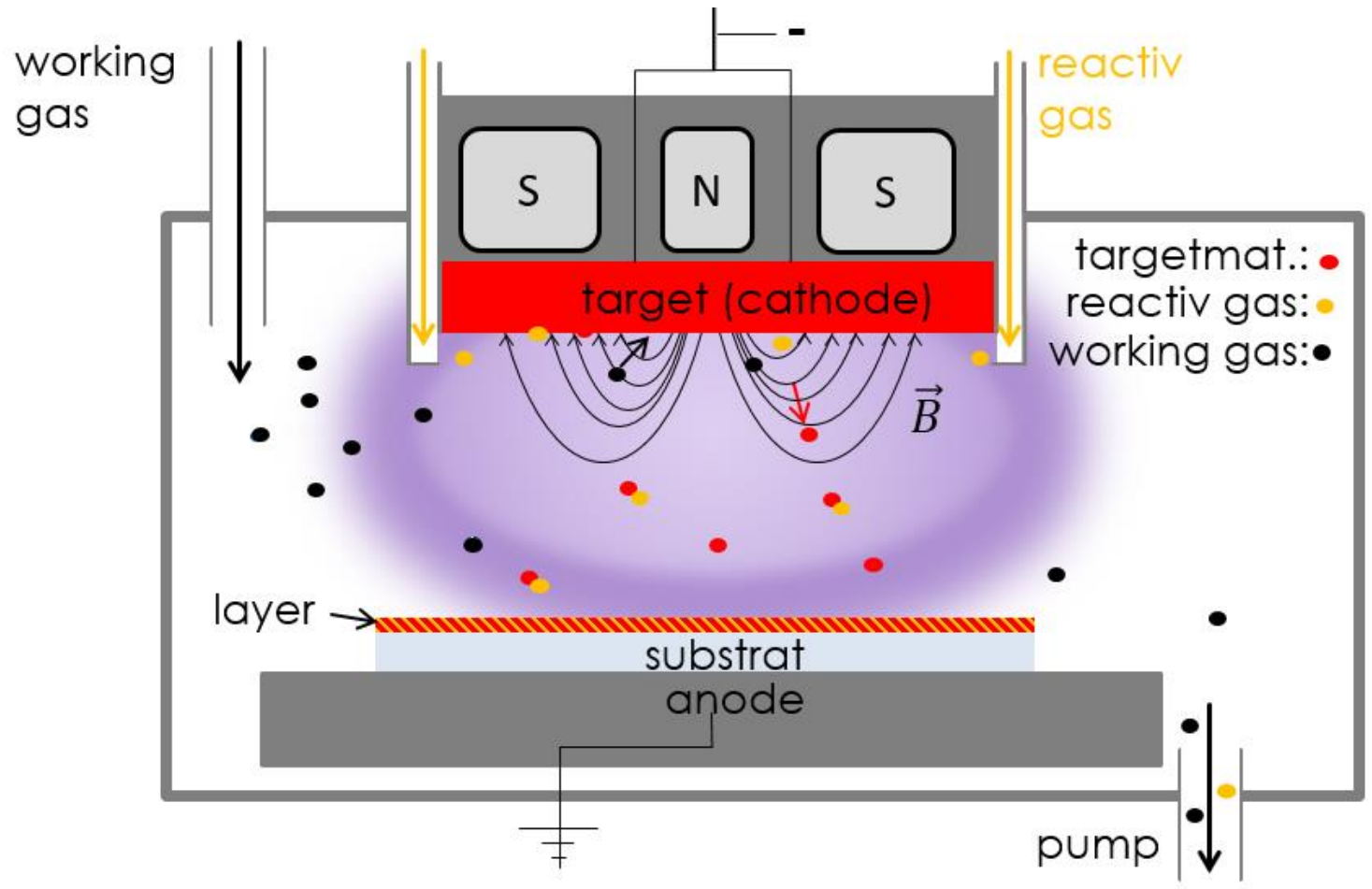




\section{Ag-based Coatings (Protection)}

Investigation of reactive sputtered $\mathrm{Al}_{2} \mathrm{O}_{3}, \mathrm{SiO}_{2}, \mathrm{Si}_{3} \mathbf{N}_{4}$

- Influence of gas supply: Influence of $\mathrm{Ar}, \mathrm{O}_{2}, \mathrm{~N}_{2}$ on resistance to aq. solutions and stress

\begin{tabular}{|c|c|c|c|c|}
\hline Layer & $\begin{array}{c}\text { Ar-supply } \\
\text { [sccm] }\end{array}$ & $\begin{array}{c}\mathrm{O}_{2} \\
\text { [operating point] }\end{array}$ & $\begin{array}{c}\mathrm{N}_{2} \text {-supply } \\
\text { [sccm] }\end{array}$ & $\begin{array}{l}\text { Dynamic deposition } \\
\text { rate }[\mathrm{nm} * \mathrm{~m} / \mathrm{min}]\end{array}$ \\
\hline $\mathrm{Al}_{2} \mathrm{O}_{3-} 20 \mathrm{Ar}$ & 20 & intermediate partial pressure & & 3 \\
\hline $\mathrm{Al}_{2} \mathrm{O}_{3-} 35 \mathrm{Ar}$ & 35 & intermediate partial pressure & & 3 \\
\hline $\mathrm{Al}_{2} \mathrm{O}_{3-} 160 \mathrm{Ar}$ & 160 & intermediate partial pressure & & 3 \\
\hline $\mathrm{SiO}_{2-} 2 \mathrm{OAr}$ & 20 & $\uparrow \mathrm{O}_{2}$ partial pressure & & 8 \\
\hline $\mathrm{SiO}_{2-} 35 \mathrm{Ar}$ & 35 & intermediate partial pressure & & 17 \\
\hline $\mathrm{SiO}_{2-160 \mathrm{Ar}}$ & 160 & intermediate partial pressure & & 19 \\
\hline $\mathrm{Si}_{3} \mathrm{~N}_{4} \_1$ & 20 & & 33 & 12 \\
\hline $\mathrm{Si}_{3} \mathrm{~N}_{4-2} 2$ & 20 & & 120 & 7 \\
\hline $\mathrm{Si}_{3} \mathrm{~N}_{4-3} 3$ & 80 & & 47 & 12 \\
\hline $\mathrm{Si}_{3} \mathrm{~N}_{4} 4$ & 80 & & 120 & 8 \\
\hline
\end{tabular}




\section{Ag-based Coatings (Protection)}

Results: Resistance to aqueous solution

- Removal rates in $\mathrm{nm} / \mathrm{h}$ for exposure to a basic solution (pH-value: 11 - 11.5):
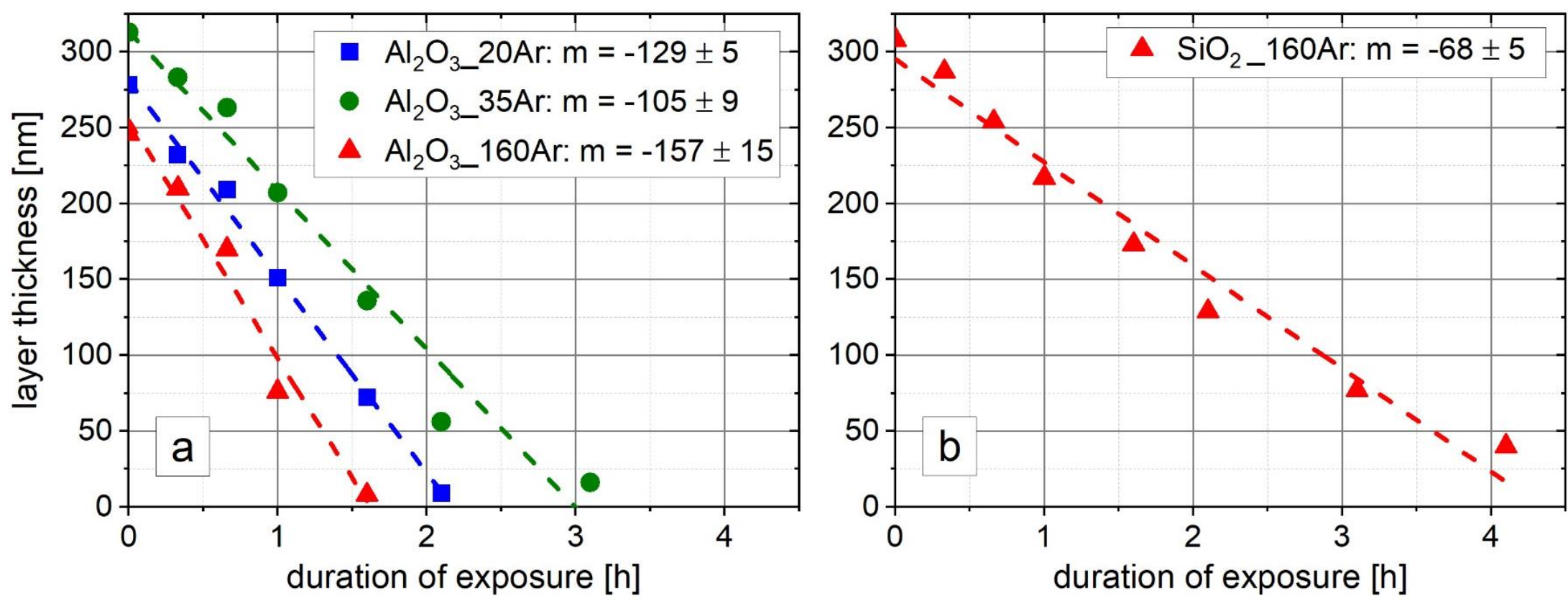


\section{Ag-based Coatings (Protection)}

Results: Removal rates in $\mathrm{nm} / \mathrm{h}$ for exposure to a basic solution ( $\mathrm{pH}$-value: 12,5 - 13)
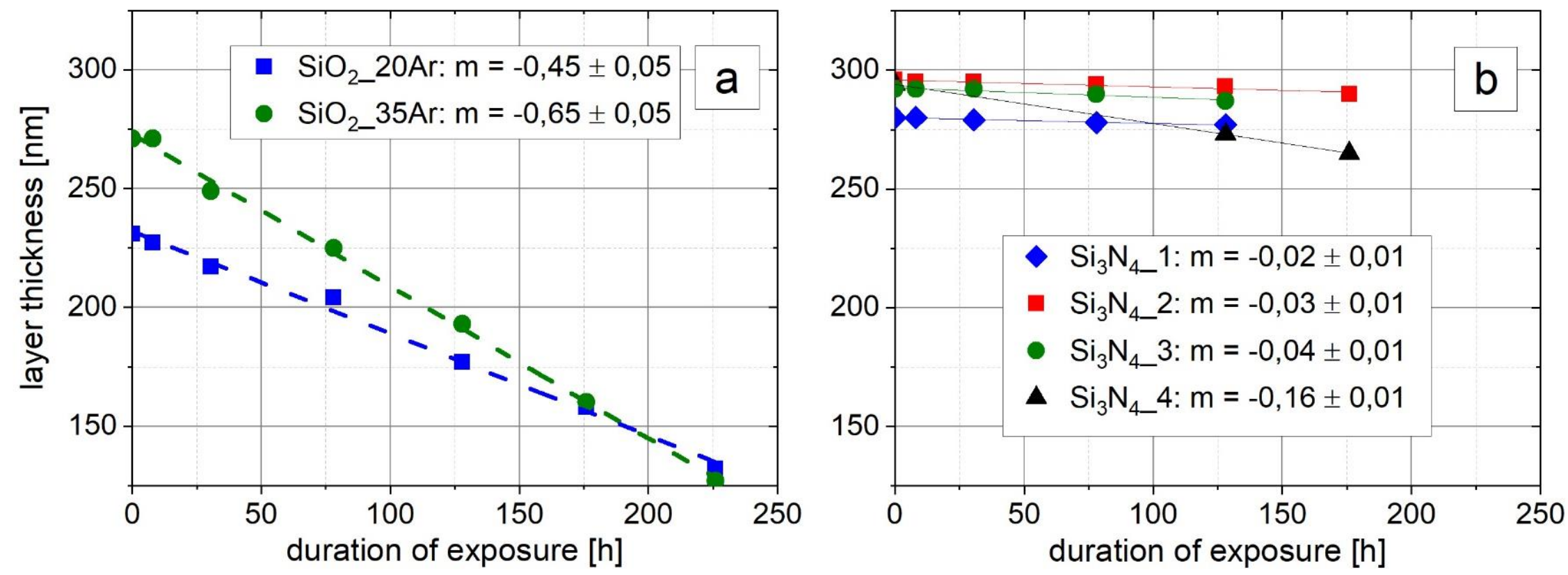

- Resistance to aq. solution: $\mathrm{Al}_{2} \mathrm{O}_{3}<\mathrm{SiO}_{2}<\mathrm{Si}_{3} \mathrm{~N}_{4}$

- Resistance to aq. solutions depends on gas supply, e.g. for $\mathrm{SiO}_{2}$ less Ar-flow is preferable 


\section{Ag-based Coatings (Protection)}
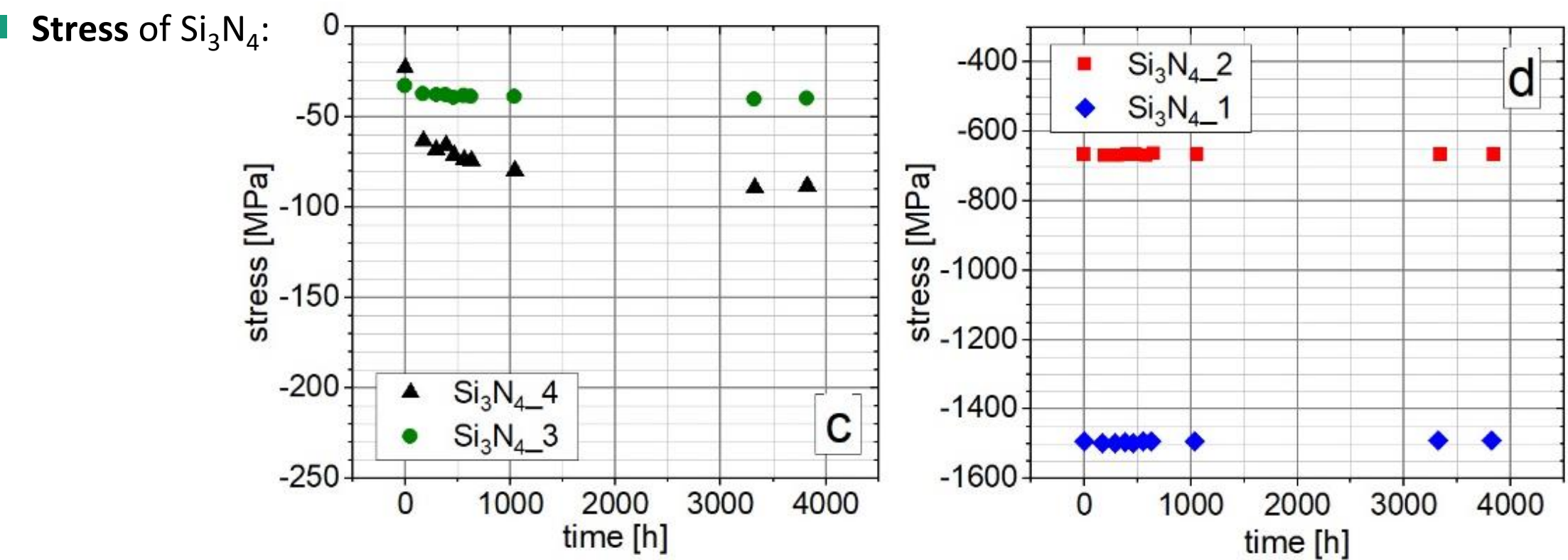

Deposition of $\mathrm{Si}_{3} \mathrm{~N}_{4}$ :

\begin{tabular}{|l|l|l|l|}
\hline Layer & Ar-supply [sccm] & $\mathbf{N}_{\mathbf{2}}$-supply [sccm] & dep. rate [nm*m/min] \\
\hline $\mathrm{Si}_{3} \mathrm{~N}_{4 \_} 1$ & 20 & 33 & 12 \\
\hline $\mathrm{Si}_{3} \mathrm{~N}_{4 \_} 2$ & 20 & 120 & 7 \\
\hline $\mathrm{Si}_{3} \mathrm{~N}_{4 \_} 3$ & 80 & 47 & 12 \\
\hline $\mathrm{Si}_{3} \mathrm{~N}_{4 \_} 4$ & 80 & 120 & 8 \\
\hline
\end{tabular}




\section{Ag-based Coatings}

Ag with a nanolaminate which contains the most stable $\mathrm{Si}_{3} \mathrm{~N}_{4}$ as a final layer was applied

- The nanolaminate acts as one optical material

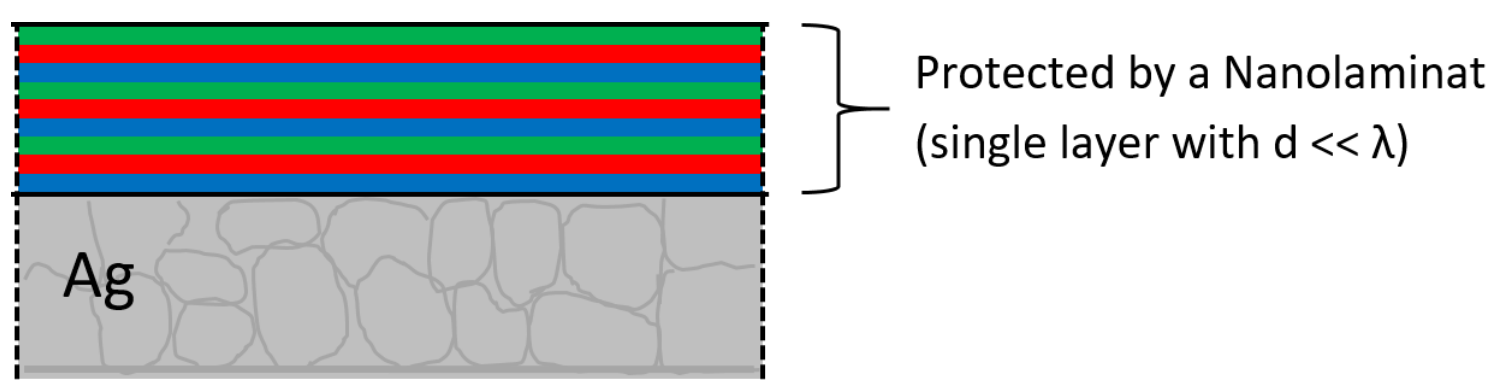




\section{Ag-based Coatings}

- Optical performance (960 - $5300 \mathrm{~nm})$ :

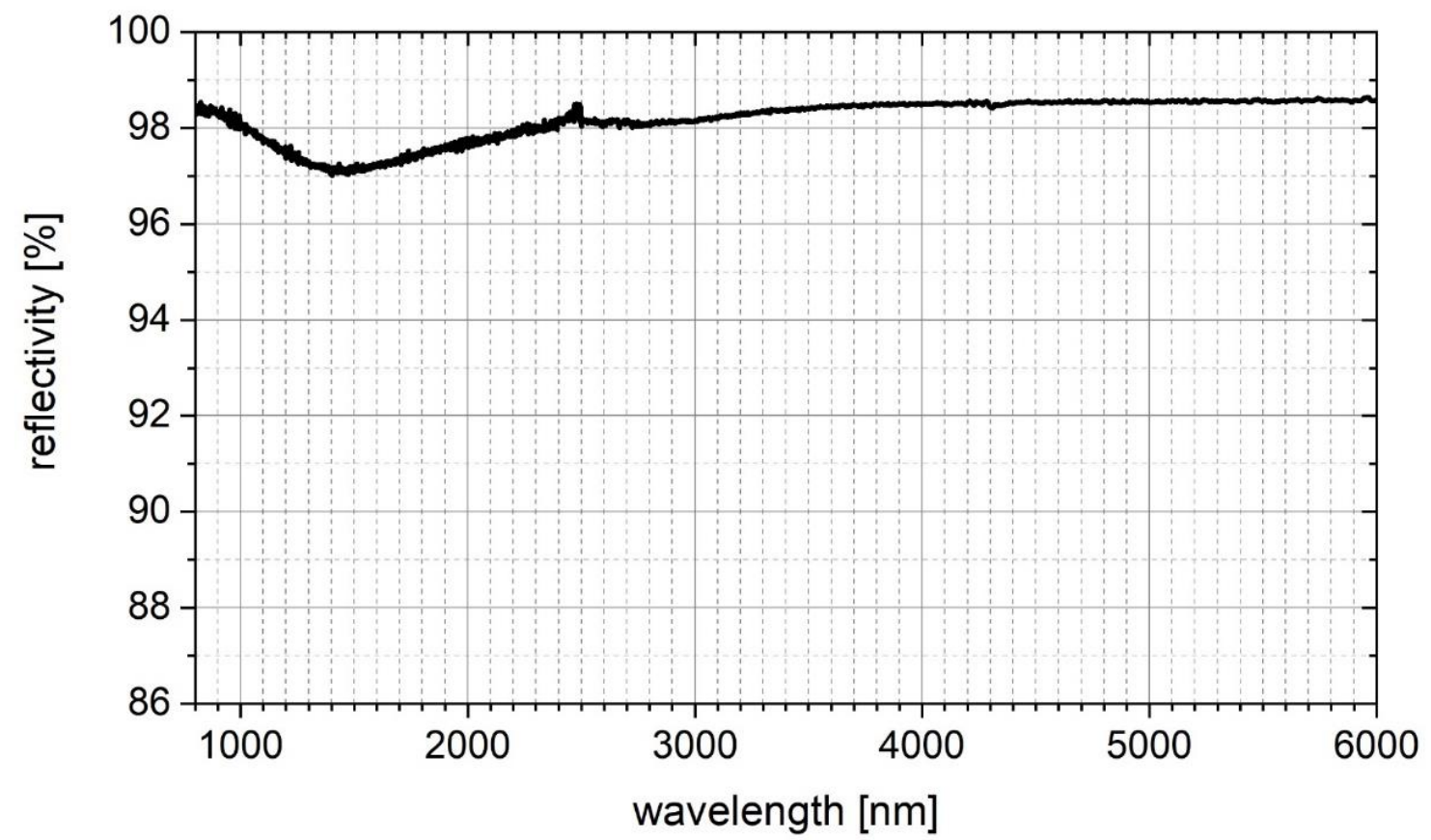

- Conclusion: - Highly durable Ag-based coating with a tailored $\mathrm{Si}_{3} \mathrm{~N}_{4}$ final layer

- Risk management: Same approach for space based application 


\section{Thank you for your attention!}

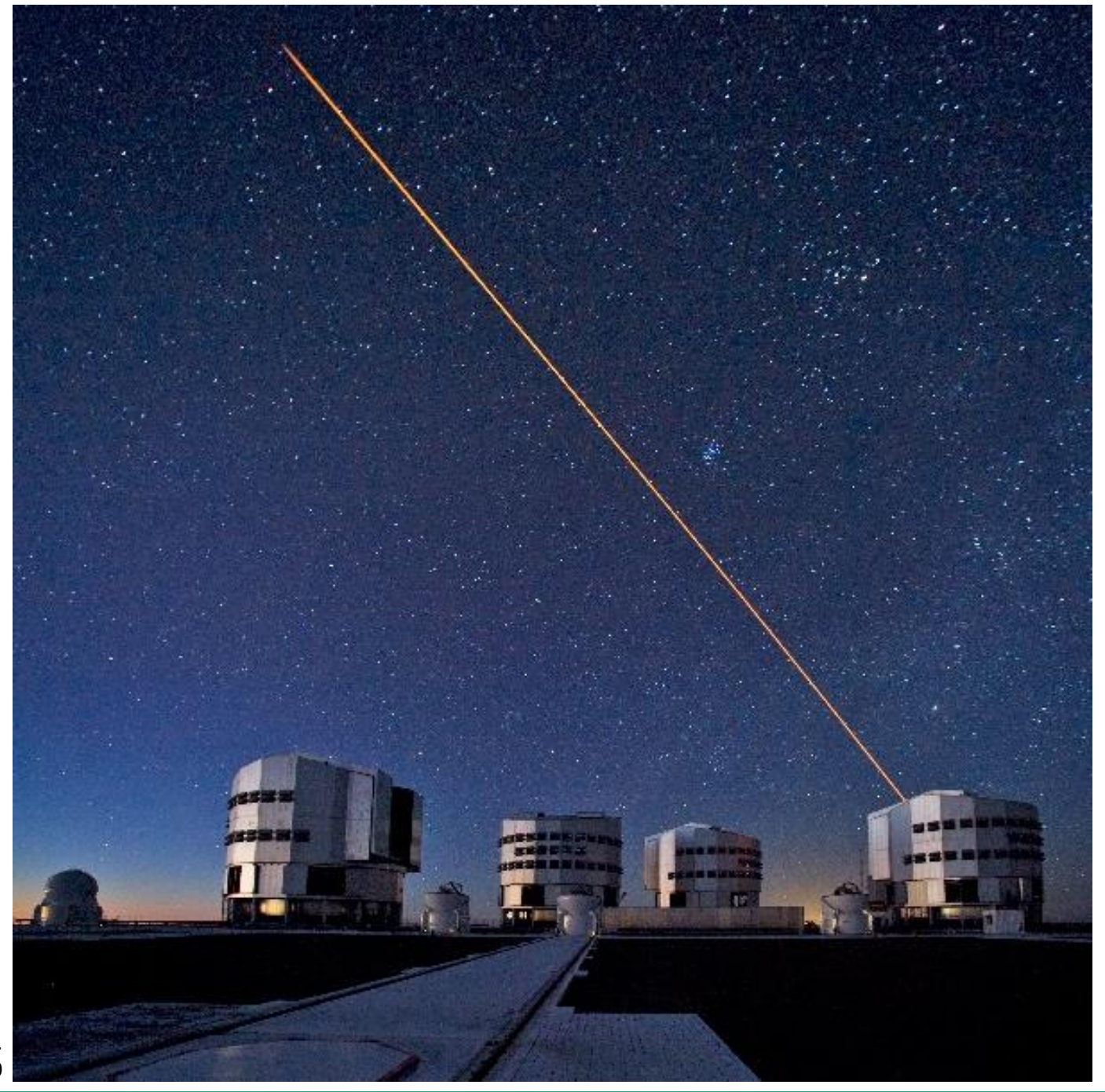



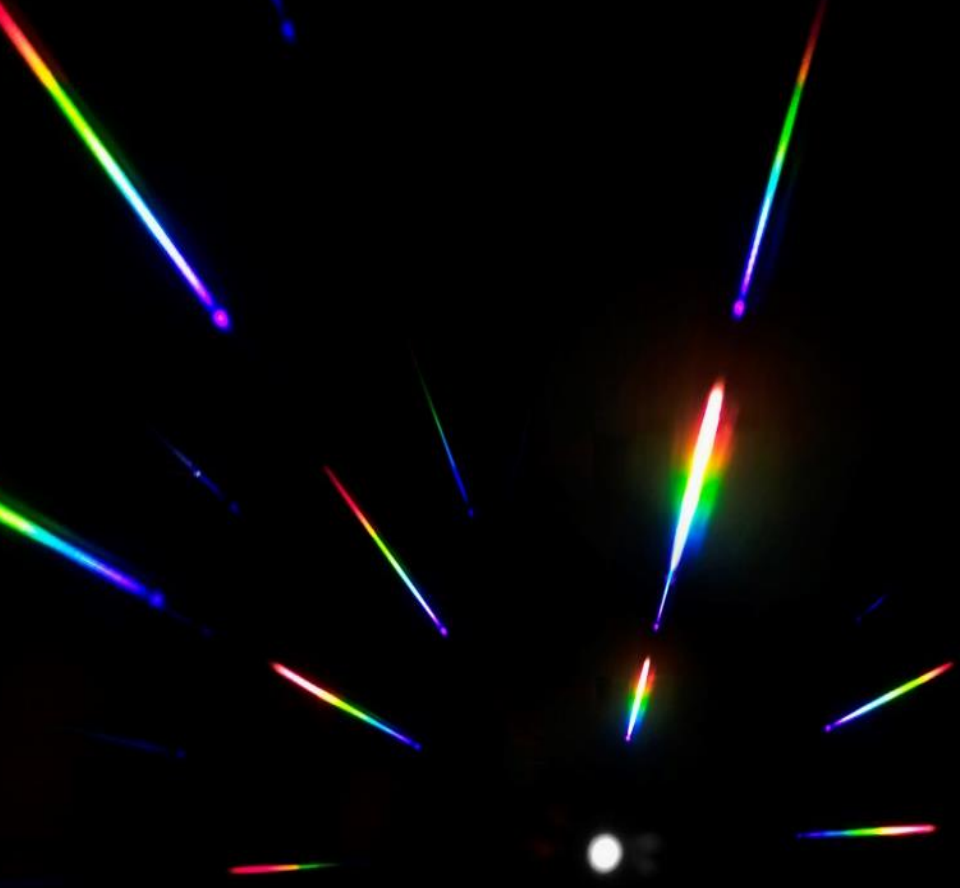

\section{Fraunhofer IOF -} Enabling Research. Empowering Innovation 


\section{Results}

intermediate partial pressure = operating point in transition region

- $\uparrow \mathrm{O}_{2}$ partial pressure = operating point in the compound region
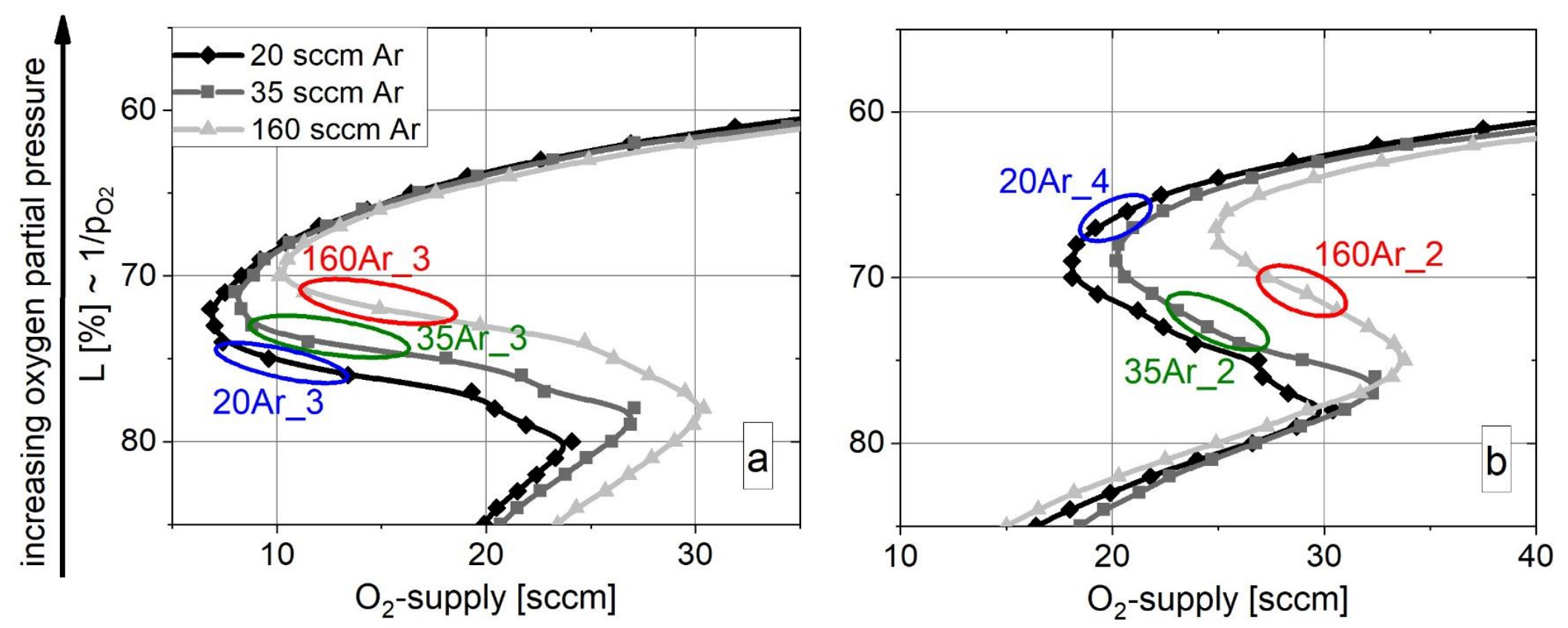


\section{Results}

\section{Stress}
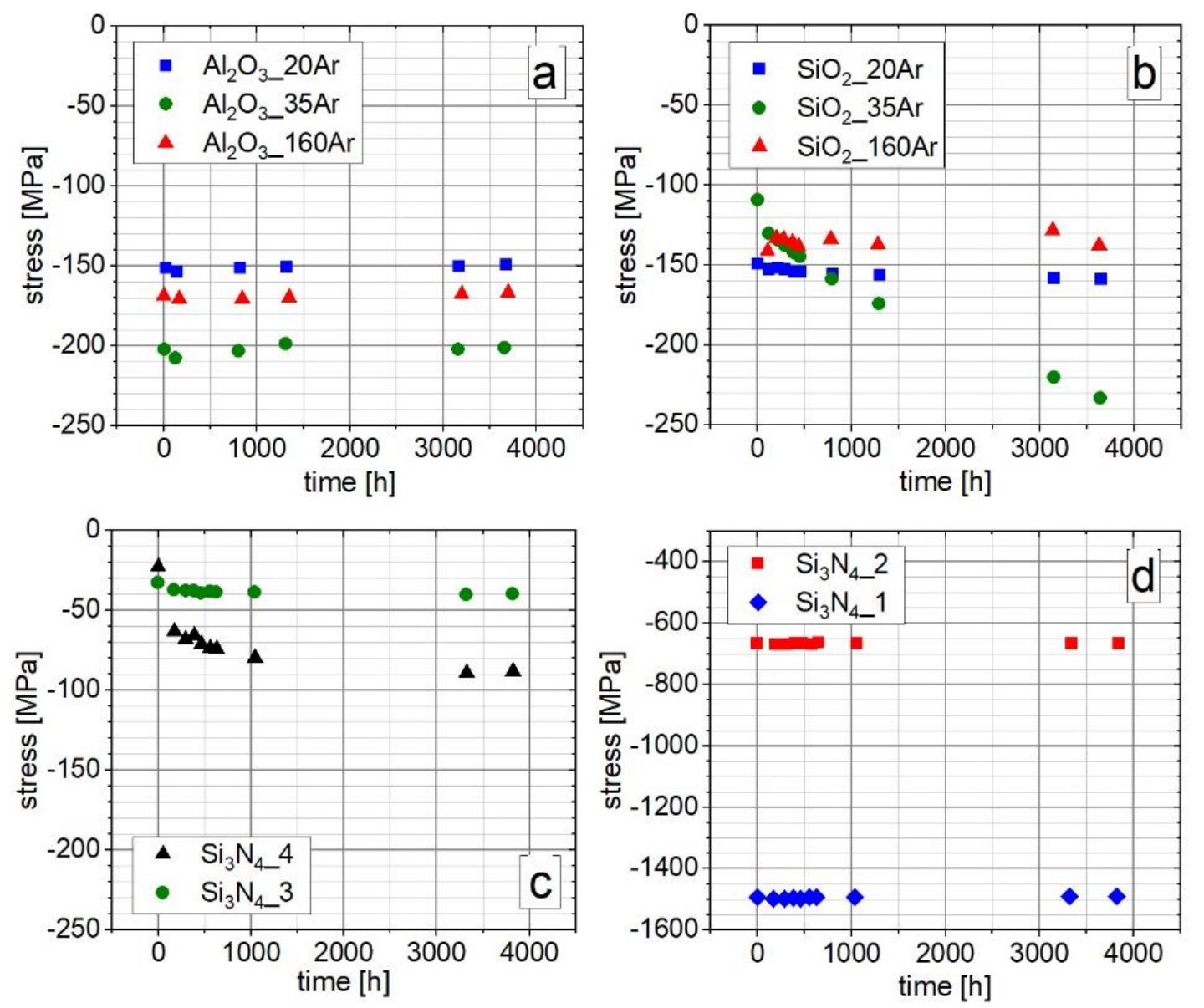